\title{
Definición Automática de la Geometría de Herramientas de Corte usando Algoritmos Genéticos
}

\author{
O. Durán ${ }^{1}$, N. Rodríguez ${ }^{2}$ y L.A. Consalter ${ }^{3}$ \\ (1) Pontificia Universidad Católica de Valparaíso, Escuela de Ingeniería Mecánica, \\ Av. Los Carrera 01567, Quilpue-Chile (e-mail: orlando.duran@ucv.cl) \\ (2) Pontificia Universidad Católica de Valparaíso, Escuela de Ingeniería Informática, Av. Brasil 2241, \\ Valparaíso-Chile (e-mail: nibaldo.rodriguez@ucv.cl) \\ (3) Universidade de Passo Fundo, FEAR, 99001-970 Passo Fundo, RS-Brasil (e-mail: lac@upf.br)
}

\section{Resumen}

Este trabajo presenta la utilización de una técnica de optimización evolutiva desarrollada en Matlab para la obtención de los ángulos principales de la geometría del filo de corte de una herramienta de mecanizado. El método de optimización se basa en el modelo analítico, no lineal de Kaldor y Venuvinod. Diferentes configuraciones del algoritmo genético fueron evaluadas en función de datos de mecanizados obtenidos de la literatura. Se desarrollaron varias simulaciones para validar el desempeño del algoritmo y mostrar la utilidad de la técnica aplicada. Se concluye que la técnica propuesta presenta estabilidad y coherencia en sus resultados a lo largo de los experimentos efectuados.

Palabras clave: geometría de corte, mecanizado, algoritmos genéticos, planificación de procesos

\section{Automatic Definition of the Cutting Tool Geometry using Genetic Algorithms}

\section{Abstract}

This paper presents the application of an evolutionary algorithm using Matlab to obtain the optimum main angles of the cutting geometry of a machining tool. The optimization method considered the non linear model of Kaldor and Venuvinod. Different algorithm configurations were tested using data obtained from the literature. Several simulations were carried out to validate the performance of the algorithm and to show the usefulness of the method employed. It is concluded that that the proposed approach shows to be stable and coherent in the results for the cases studied.

Keywords: cutting tool geometry, machining, genetic algorithm, process planning 


\section{INTRODUCCIÓN}

La Planificación de Procesos de Fabricación es una actividad que está dedicada a la definición de todas las instrucciones y parámetros necesarios para fabricar un determinado producto o componente. La planificación de procesos asistida por computador (Computer aided process planning, CAPP) es considerada la implementación tecnológica de sistemas de apoyo a la actividad de planificación de procesos. Durante estas actividades se definen entre otros, la secuencia de actividades, los parámetros, máquinas, dispositivos y accesorios requeridos, además de estimarse los tiempos y los costos del proceso de fabricación planificado (Kalpakjian y Schmid, 2002; Groover, 1997; Halevi y Weill, 1995). En la literatura se pueden encontrar diversos trabajos que apuntan a la determinación de la secuencia de operaciones, definición de parámetros de corte y estimación de tiempos y costos. Sin embargo, un aspecto de gran importancia para la eficiencia de estas operaciones, como es la definición automática de la geometría de corte de la herramienta, no ha sido abordada por las investigaciones hasta el presente momento.

Un algoritmo genético (AG), es una técnica de la Inteligencia Artificial que imita a la evolución biológica como estrategia para resolver problemas (Holland, 1992). Ellos forman parte de los llamados Algoritmos Evolutivos (AE). Dado un problema específico a resolver, la entrada del A.G. es un conjunto de soluciones potenciales a ese problema, codificadas de alguna manera, y que permite evaluar cuantitativamente a cada opción. Luego el AG evalúa cada candidata de acuerdo con la función de aptitud (fitness). En un conjunto de candidatas generadas aleatoriamente, algunas no funcionarán en absoluto, y serán eliminadas, pero habrá otras que pueden ser prometedoras hacia la solución del problema. Estas candidatas prometedoras se conservan y se realizan múltiples copias de ellas, pero las copias no son perfectas, sino que se introducen cambios aleatorios (operaciones de cruzamiento y mutación) durante el proceso de copia, formando un nuevo conjunto de soluciones candidatas, siendo sometidas a una nueva ronda de evaluación de aptitud. Las candidatas que han empeorado o no han mejorado con los cambios en su código son eliminadas; pero, el subconjunto de la población que vio mejorada su aptitud es considerado como el de mejores soluciones del problema, más completas o más eficientes. De nuevo, se seleccionan y copian estos individuos vencedores hacia la siguiente generación con cambios aleatorios y el proceso se repite hasta que una cierta condición de término se alcance. Los algoritmos genéticos han demostrado ser una estrategia enormemente poderosa y exitosa para resolver problemas de optimización.

Existe en la literatura un número importante de aplicaciones de los algoritmos evolutivos en problemas de manufactura. Dereli y Filiz (1999), proponen el uso de A.G. para la determinación automática de la mejor organización de herramientas a partir de la representación en 3 dimensiones de una pieza a fabricar. McMullen et al. (2003), se han focalizado en la optimización de secuencias de fabricación a través de la definición de un límite de cambios de herramientas durante el ciclo de fabricación de un dado componente. Habel y Slodky (2006), desarrollaron un sistema especialista que se integra a un sistema de planificación de procesos para la configuración de herramientas de corte. Quiza et al. (2006), presentan un mecanismo de optimización multi-objetivo utilizando AG para la determinación de un conjunto de parámetros de corte en operaciones de torneado.

Específicamente, la sección de herramientas de corte ha sido explorada más recientemente por investigadores. Es así como Oral y Cakir (2004), presentan un sistema para la selección automática de herramientas de corte. La selección es ejecutada usando datos de maquinabilidad, datos de la máquina herramienta, información sobre la forma de sujeción de la pieza y el numero de herramientas disponibles. La selección de las herramientas esta enfocado hacia la definición de una secuencia optima de herramientas, esto es aquella que minimice el número de cambios de herramientas necesarios y los tiempos de desplazamientos de las mismas. Toussaint y Cheng (2006), proponen un sistema basado en la web para auxiliar en la selección de herramientas de corte. Este sistema se basa en la tecnología de razonamiento a través de casos. Zhao et al.(2002), proponen un sistema basado en conocimiento que realiza la selección de las herramientas y de las condiciones de corte para operaciones de torneado. El sistema, llamado CADEXCATS usa como entrada información proveniente de un sistema CAD y, a través de la aplicación de una base de reglas este determina los detalles de preparación de las herramientas. Este sistema también focaliza 
su selección hacia la minimización de herramientas en función de la geometría de la pieza a ser mecanizada.

Los procedimientos para la selección de herramientas reportados hasta ahora, usan sistemas expertos que se basan en conceptos tales como productividad, accesibilidad y otros, pero ninguno considera el impacto que la configuración del filo de corte puede causar en la vida de la herramienta, maquinabilidad, acabado superficial, quebrado de la viruta y/o en la temperatura de corte. Menos aun, no existen trabajos que hayan utilizado mecanismos o algoritmos evolutivos para estos fines. Este artículo está destinado a presentar la utilización de algoritmos genéticos como medio para la selección automática de los ángulos de herramientas de corte en un escenario de planificación de procesos auxiliado por computador.

\section{MODELO DE OPTIMIZACIÓN}

Como ya ha sido comentado, la selección de la geometría de corte de una herramienta de mecanizado es una tarea de alta relevancia y complejidad. La relevancia se basa en que las diferentes geometrías pueden provocar efectos importantes en la eficiencia y calidad de las operaciones de mecanizado. La complejidad es atribuible a la gran cantidad de factores y alternativas que existe entre las herramientas de corte. Gran oferta de herramientas de corte existen en el mercado. De esta forma, los planificadores se ven obligados a trabajar con voluminosos manuales de mecanizado y catálogos de herramientas. Además, las reglas para la definición de la geometría del filo cortante suelen ser vagas, aproximadas y en cierta forma contradictorias. Por lo tanto, resulta prácticamente imposible a los especialistas verificar todas las alternativas desde el punto de vista de su efectividad y potencial. Una salida es la definición de modelos de optimización para la obtención de la geometría más apropiada. Kaldor y Venuvinod (1997), han definido un modelo para la macro optimización de la geometría de corte en herramientas de geometría definida. El modelo propuesto relaciona el material de la pieza, material de la herramienta y la geometría del filo cortante. Ellos proponen un número llamado $\sigma_{r}$, que caracteriza el conjunto ideal de ángulos de la herramienta. El criterio de optimización está basado en la maximización de la vida de la herramienta. La ecuación 1 muestra este modelo.

$\sigma_{r}=\cos ^{2} \lambda_{s} \frac{\operatorname{sen}\left(\alpha_{n}+\frac{\beta_{n}}{2}\right) \operatorname{sen}\left(\frac{\beta_{n}}{2}\right)\left(\frac{\beta_{n}}{2}+\frac{1}{2} \operatorname{sen} \beta_{n}\right)-\cos \left(\alpha_{n}+\frac{\beta_{n}}{2}\right) \cos \left(\frac{\beta_{n}}{2}\right)\left(\frac{\beta_{n}}{2}-\frac{1}{2} \operatorname{sen} \beta_{n}\right)}{\left(\frac{\beta_{n}}{2}\right)^{2}-\left(\frac{1}{2} \operatorname{sen} \beta_{n}\right)^{2}}$

Donde los ángulos usados por el modelo corresponden a los definidos por la norma ISO 3002/1 que se representa en la figura 1 .

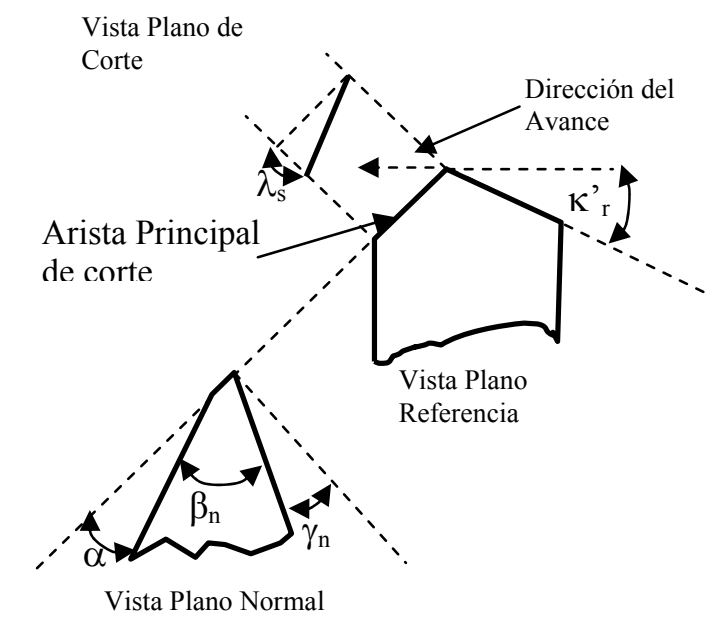

Fig 1: Geometría del filo cortante según la norma ISO 3002/1. 
Las figuras $2 a$ y $2 b$ muestran las relaciones que existen entre los ángulos alfa y lambda y el valor de $\sigma_{\mathrm{r}} \mathrm{y}$, alfa y gamma y el valor de $\sigma_{\mathrm{r}}$, respectivamente.

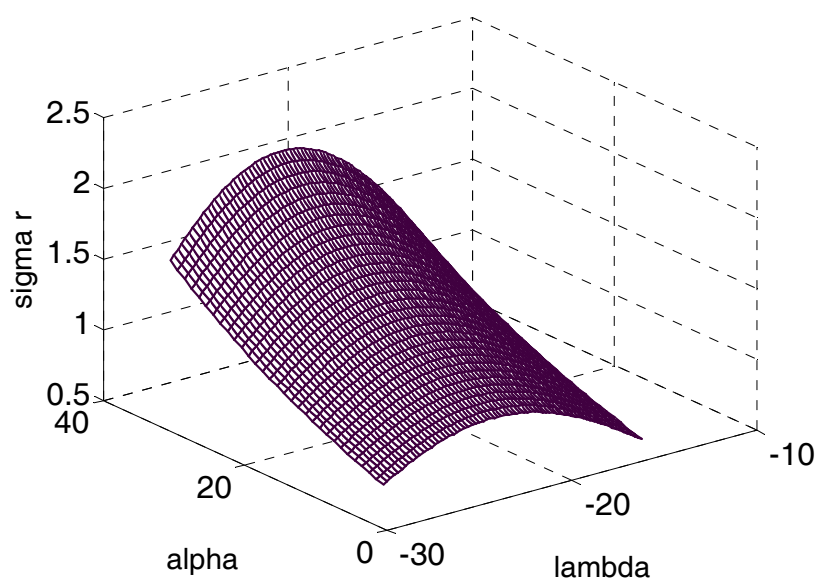

a)

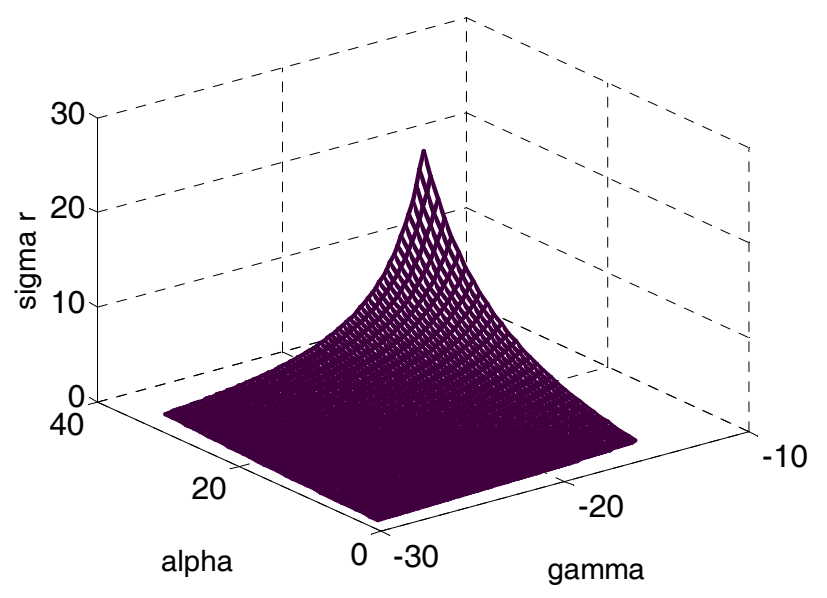

b)

Fig 2: Relaciones entre los ángulos del filo de corte y el valor de $\sigma_{\mathrm{r}}$ a) alfa ,lambda versus $\sigma_{r}$. b) alfa, gamma versus $\sigma_{r}$

Por otro lado, el número $G$ representa la relación ideal entre el material de la herramienta y el material de la pieza. $G$ ha sido obtenido de manera empírica y, según los autores, esta relación maximiza la durabilidad de la herramienta desde el punto de vista de la resistencia al desgaste y menor propensión a la fractura del filo de corte. La relación se muestra en la ecuación 2.

$\mathrm{G}=\log _{10}\left\{\mathrm{Sb} /\left(\mathrm{EU}^{2}\right)\right\}+4.73$

Donde Sb representa la tensión a la flexión del material de la herramienta, E el módulo de elasticidad del mismo y U la presión específica de corte del material de la pieza (todos estos tres parámetros se expresan en $\mathrm{GPa}$ ). El valor 4.73 representa un factor de corrección y es adimensional. Para mayores detalles de la obtención de esta expresión remitirse a Kaldor y Venuvinod (1997). En consecuencia, la geometría ideal para una dada situación es la que minimiza la diferencia entre $\mathrm{G}$ y $\sigma_{\mathrm{r}}$.

\section{METODOLOGÍA}

Como ya fue comentado, se pretende minimizar la diferencia entre $\sigma_{r}$ y G. Esto sujeto a las restricciones relacionadas con las dimensiones usuales de los ángulos del filo de corte.

$\operatorname{Min} f(\lambda, \alpha, Y)=\operatorname{ABS}\left(\sigma_{r}-G\right)$

Sujeto a:

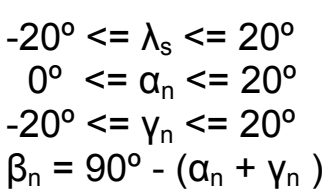

Para obtener los parámetros más adecuados de los algoritmos genéticos se realizó una serie de pruebas. Para estas se utilizaron los datos reportados por Kaldor y Venuvinod (1997), y que se resumen en la tabla 1. Las pruebas realizadas consisten en la variación de los siguientes parámetros: probabilidad de cruzamiento, tasa de mutación llegando a 24 combinaciones de estos valores. En cada corrida de prueba se registró el error cuadrático medio cuyo valor medio después de 20 corridas para cada una de las 38 situaciones y cada una de las 24 combinaciones de parámetros del A.G. se 
muestra en la tabla 3. También se registró el número promedio en el cual se alcanzó la condición de término (error menor a $10^{-6}$ ó 100 iteraciones, lo que ocurra primero).

Tabla 1: Datos experimentales usados para comparación de los parámetros del A.G.

\begin{tabular}{|c|c|c|c|}
\hline$\alpha$ & $\gamma$ & $\lambda$ & $\sigma$ \\
\hline 10 & -4 & -8 & 1,82 \\
\hline 18 & -18 & -16 & 1,82 \\
\hline 6,2 & -16 & -20 & 1,25 \\
\hline 0 & -3 & 0 & 1,32 \\
\hline 1 & 0 & 0 & 1,4 \\
\hline 0 & 3 & 0 & 1,41 \\
\hline 5 & $-7,5$ & 0 & 1,5 \\
\hline 4,8 & $-6,1$ & $-3,5$ & 1,51 \\
\hline 4,8 & $-6,1$ & $-3,5$ & 1,51 \\
\hline 6 & -6 & 6 & 1,56 \\
\hline 4 & 0 & 0 & 1,58 \\
\hline 3,5 & 3,5 & 3,5 & 1,61 \\
\hline 10 & -10 & -13 & 1,62 \\
\hline 9,8 & -12 & -7 & 1,63 \\
\hline 6 & 0 & 0 & 1,7 \\
\hline 4,8 & 4,8 & $-1,3$ & 1,73 \\
\hline 8 & 15 & 0 & 2,34 \\
\hline 8,9 & 18 & $-1,3$ & 2,34 \\
\hline 8 & 8 & 0 & 207 \\
\hline
\end{tabular}

\begin{tabular}{|c|c|c|c|}
\hline$\alpha$ & $\gamma$ & $\lambda$ & $\sigma$ \\
\hline 10 & 16 & 0 & 2,82 \\
\hline 13 & 15 & 0 & 2,96 \\
\hline 5 & 8 & 0 & 1,83 \\
\hline 4,8 & 9,6 & $-2,6$ & 1,85 \\
\hline 4,8 & 9,7 & $-2,6$ & 1,86 \\
\hline 4 & 15 & 0 & 1,94 \\
\hline 4,8 & 14 & 6,6 & 1,96 \\
\hline 4,8 & 14 & 6,6 & 1,98 \\
\hline 6 & 14 & 6 & 2,08 \\
\hline 8 & 9,8 & 5,7 & 2,11 \\
\hline 7,9 & 10,4 & 4,1 & 2,14 \\
\hline 5,8 & 15,5 & 4,1 & 2,18 \\
\hline 7,9 & 16,8 & 2,3 & 2,35 \\
\hline 10,4 & 15,8 & 18,7 & 2,39 \\
\hline 4,8 & 4,8 & $-1,3$ & 1,73 \\
\hline 7 & $-0,7$ & -4 & 1,74 \\
\hline 5 & 6 & 0 & 1,78 \\
\hline 8 & 24 & 4 & 2,79 \\
\hline 13 & 5 & 0 & 2,42 \\
\hline
\end{tabular}

Tabla 2: Detalles de los parámetros del AG usados en 24 experimentos

\begin{tabular}{ccc|ccc}
\hline $\begin{array}{c}\text { Número de } \\
\text { la Prueba }\end{array}$ & $\begin{array}{c}\text { Probabilidad } \\
\text { Cruzamiento }\end{array}$ & $\begin{array}{c}\text { Tasa de } \\
\text { Mutación }\end{array}$ & $\begin{array}{c}\text { Número de } \\
\text { la Prueba }\end{array}$ & $\begin{array}{c}\text { Probabilidad } \\
\text { Cruzamiento }\end{array}$ & $\begin{array}{c}\text { Tasa de } \\
\text { Mutación }\end{array}$ \\
\hline 1 & 0.1 & 0.1 & 13 & 0.5 & 0.5 \\
2 & 0.3 & 0.1 & 14 & 0.7 & 0.5 \\
3 & 0.5 & 0.1 & 15 & 0.9 & 0.5 \\
4 & 0.7 & 0.1 & 16 & 0.1 & 0.7 \\
5 & 0.9 & 0.1 & 17 & 0.3 & 0.7 \\
6 & 0.1 & 0.3 & 18 & 0.5 & 0.7 \\
7 & 0.3 & 0.3 & 19 & 0.7 & 0.7 \\
8 & 0.5 & 0.3 & 20 & 0.9 & 0.7 \\
9 & 0.7 & 0.3 & 21 & 0.1 & 0.9 \\
10 & 0.9 & 0.3 & 22 & 0.3 & 0.9 \\
11 & 0.1 & 0.5 & 23 & 0.5 & 0.9 \\
12 & 0.3 & 0.5 & 24 & 0.7 & 0.9 \\
\hline
\end{tabular}

Los parámetros seleccionados para este algoritmo genético son los siguientes:

Probabilidad de Cruzamiento $=0.7$

Tasa de Mutación= 0.3

La figura 3 muestra como este algoritmo genético convergió hacia una solución satisfactoria usando los parámetros seleccionados y mostrados arriba. 
Tabla 3: Promedio de los errores obtenidos en los 24 experimentos realizados

\begin{tabular}{lrlr}
\hline No. del Exper. & Error Cuad. Medio & No. del Experim. & Error Cuad. Medio \\
\hline 1 & $7,72 \mathrm{E}-06$ & 13 & $6,57 \mathrm{E}-06$ \\
2 & $7,19 \mathrm{E}-06$ & 14 & $4,93 \mathrm{E}-06$ \\
3 & $8,70 \mathrm{E}-06$ & 15 & $8,54 \mathrm{E}-06$ \\
4 & $1,10 \mathrm{E}-02$ & 16 & $1,76 \mathrm{E}-05$ \\
5 & $8,10 \mathrm{E}-02$ & 17 & $7,30 \mathrm{E}-06$ \\
6 & $6,31 \mathrm{E}-06$ & 18 & $8,96 \mathrm{E}-06$ \\
7 & $6,12 \mathrm{E}-06$ & 19 & $7,90 \mathrm{E}-06$ \\
8 & $5,04 \mathrm{E}-06$ & 20 & $7,99 \mathrm{E}-06$ \\
9 & $4,33 \mathrm{E}-06$ & 21 & $3,17 \mathrm{E}-05$ \\
10 & $1,25 \mathrm{E}-05$ & 22 & $1,18 \mathrm{E}-05$ \\
11 & $1,92 \mathrm{E}-05$ & 23 & $6,26 \mathrm{E}-06$ \\
12 & $8,31 \mathrm{E}-06$ & 24 & $5,74 \mathrm{E}-06$ \\
\hline
\end{tabular}

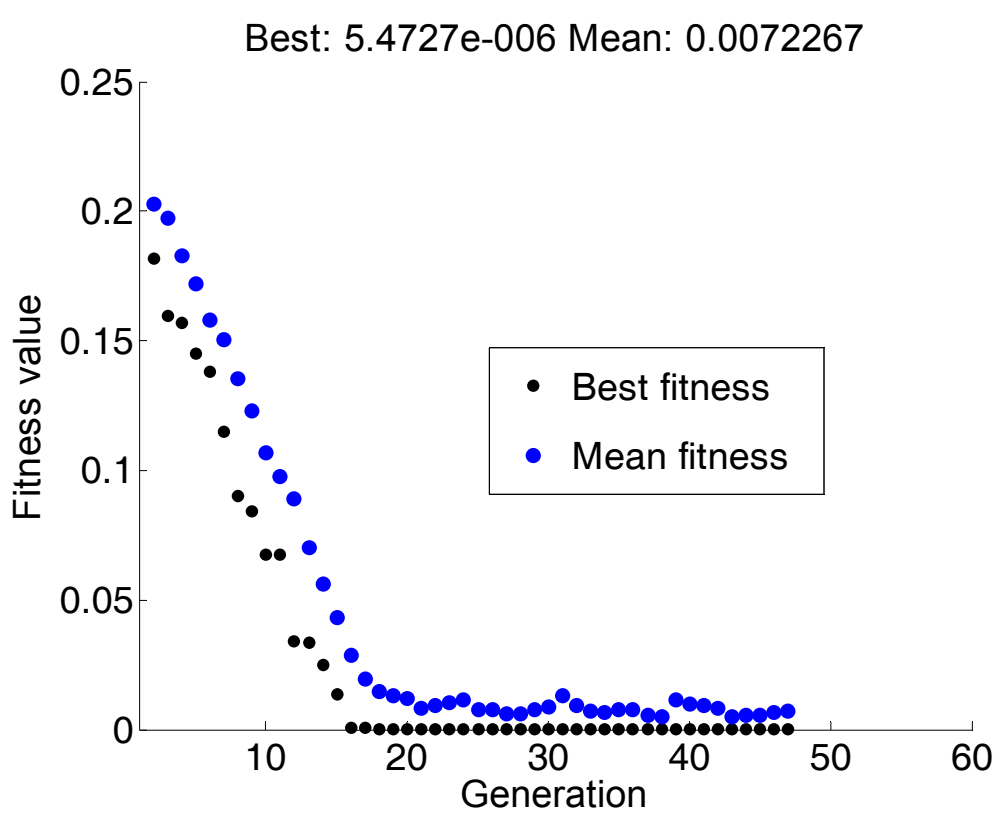

Fig. 3: Convergencia del Algoritmo con los parámetros seleccionados

\section{RESULTADOS Y DISCUSIÓN}

Para probar el enfoque propuesto en relación a su capacidad para seleccionar herramientas de corte adecuadas, se realizó un conjunto de cinco pruebas y comparaciones que se detallan a seguir. La información reunida en la literatura junto con algunos estudios de casos entregaron algunas recomendaciones de la geometría adecuada para el mecanizado de ciertos materiales usando diversos materiales de herramienta. Para cada una de las cinco comparaciones realizadas se ejecutaron 15 corridas del algoritmo genético (con los parámetros definidos en la sección anterior) obteniéndose los tres ángulos (valores promedio de las 15 corridas) para luego compararlos con los valores recomendados en la literatura. Además se obtuvo el valor del error medio absoluto (MAD, Mean Absolute Deviation) y los valores de la desviación estándar de los valores obtenidos en la 15 corridas del algoritmo para cada caso. Todos estos valores se resumen en la tabla 4.

Como se puede apreciar en los resultados los errores obtenidos para los ángulos alfa y gama pueden ser considerados como pequeños, no así el error acumulado por las estimaciones hechas 
para el ángulo lambda. A juicio de los autores, esto es de menor importancia pues, el ángulo lambda no ejerce un gran impacto en lo que respecta a la vida de la herramienta (Stemmer, 1995). Además, y como puede apreciarse en las figuras $5 a$ y $5 b$, donde se muestran las relaciones entre la función objetivo $f(\lambda, \alpha, \gamma)$ y los ángulos $\alpha_{n} y \gamma_{n}$ para dos diferentes valores de los valores de $\lambda$, se aprecia que la función es poco sensible a las variaciones de $\lambda_{s}$, lo que puede explicar en parte los errores obtenidos en la obtención de este valor. Por otro lado, las desviaciones estándar pueden ser consideradas también como pequeñas, lo que refleja una pequeña variabilidad de los resultados arrojados por el algoritmo genético. Esto último demuestra la gran estabilidad del algoritmo y de sus valores de salida.

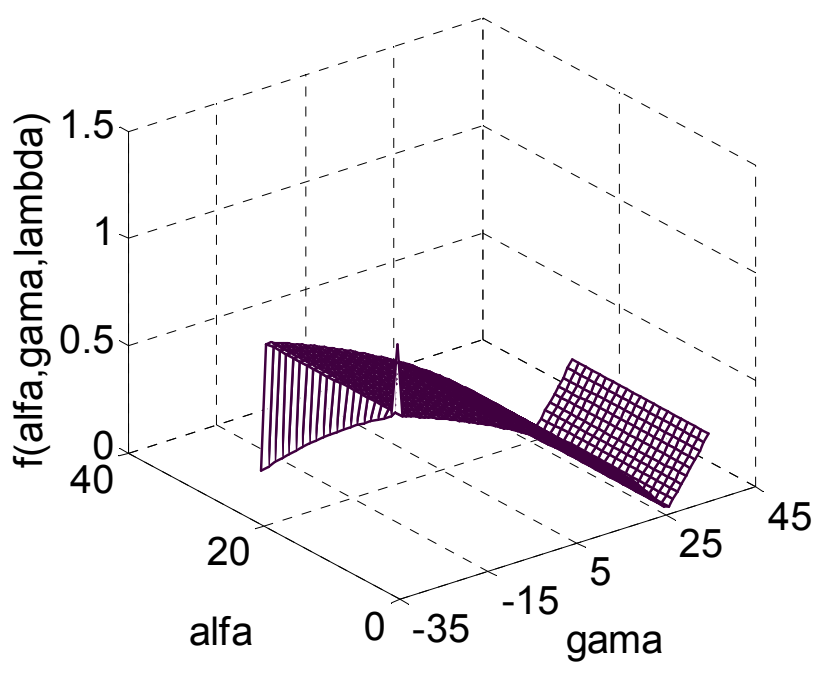

(a)

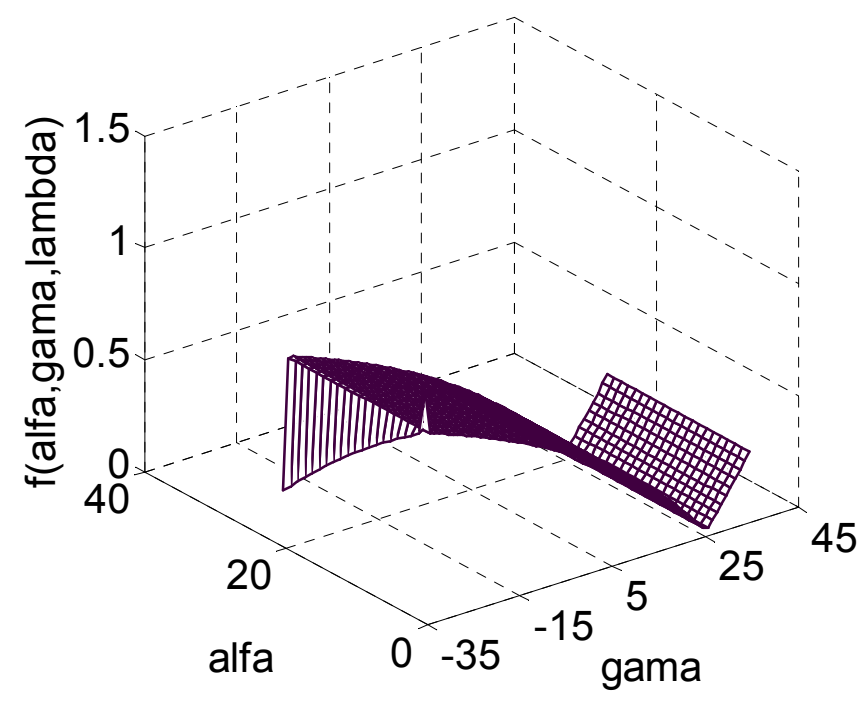

(b)

Fig. 4: Variación de la función objetivo para dos valores del ángulo de inclinación $\lambda \mathrm{s}$ en torneado de Acero AISI 1045 con herramienta de Metal Duro ISO P20. a) $35^{\circ}$; b) -25

Por último, se puede apreciar en los valores usados como estudios de casos, que el valor de los ángulos ideales del filo de corte, no es una materia cerrada, en el sentido de existir valores definidos para cada tipo de aplicación y cierto par herramienta-pieza. Lamentablemente, el único modelo cuantitativo que permite evaluar una dada geometría es el propuesto por Kaldor y Venuvinod (1997) lo que hace difícil encontrar métodos confiables de comparación para los valores obtenidos por el algoritmo genético utilizado en este trabajo.

Tabla 4: Resultados obtenidos en los estudios de caso.

\begin{tabular}{|c|c|c|c|c|c|c|c|c|c|c|c|c|c|}
\hline \multirow[b]{2}{*}{ Material de la Pieza } & \multirow[b]{2}{*}{ Material de la $\mathrm{Hta}$. } & \multicolumn{3}{|c|}{ Valores recomendados } & \multicolumn{3}{|c|}{ Valores Obtenidos } & \multicolumn{3}{|c|}{ Desviac. Standard } & \multicolumn{3}{|c|}{ Error Medio Absoluto } \\
\hline & & alfa & gama & lambda & alfa & gama & lambda & alfa & gama & lambda & alfa & gama & lambda \\
\hline Aluminio Blando & HSS M2 & 10,0 & 35,0 & 0,0 & 11,4 & 33,4 & 4,4 & 1,7 & 3,9 & 4,4 & 1,6 & 3,2 & 10,9 \\
\hline Cobre & HSS M2 & 10,6 & 19,4 & 7,5 & 10,1 & 22,7 & 13,9 & 2,4 & 5,6 & 2,6 & 1,9 & 4,7 & 6,4 \\
\hline AISI 1045 & ISO P30 & 10,0 & $-4,0$ & $-8,0$ & 5,0 & 12,3 & 13,7 & 2,4 & 7,0 & 3,9 & 5,0 & 16,3 & 21,7 \\
\hline Madera & HSS M2 & 15,0 & 40,0 & 0,0 & 14,3 & 42,2 & 11,4 & 0,4 & 0,9 & 4,4 & 0,7 & 2,2 & 11,4 \\
\hline Cobre & HSS M2 & 10,0 & 30,0 & 0,0 & 10,8 & 29,9 & 10,9 & 1,9 & 4,6 & 3,9 & 1,7 & 3,6 & 10,9 \\
\hline & & & & & & Valore & Medios & 1,76 & 4,4 & 3,8 & 2,2 & 6,0 & 12,3 \\
\hline
\end{tabular}




\section{CONCLUSIONES}

Se ha aplicado un enfoque evolutivo al proceso de obtención automática de los ángulos principales del filo de corte de una herramienta de torneado. El enfoque se basa en la utilización de algoritmos genéticos a un modelo de optimización basado en las ecuaciones obtenidas empíricamente por Kaldor y Venuvinod. Se desarrollaron experimentos para obtener la mejor combinación de parámetros del algoritmo. Toda la implementación fue realizada utilizando el software Matlab versión 7.0. Como trabajos futuros se pretende integrar esta herramienta a un software CAM, además de probar otras estrategias de optimización como meta heurísticas considerando estrategias híbridas.

\section{REFERENCIAS}

Dereli, T.R. e I.H.S. Filiz; Optimisation of process planning functions by genetic algorithms. Int. J. Comput. Ind. Eng.: 36(2),281-308 (1999).

Groover, M.P.; Fundamentos de manufactura moderna: materiales, procesos y sistemas. 973-975, Prentice-Hall Hispanoamericana. Ciudad de México. México (1997).

Habel, J y B. Slodky; The method of automated tool and cutting parameters selection for CAPP utilization.Int.Jour.Adv. Mfg. Tech., 29(1-2), 24-34 (2006).

Halevi, G.y R. Weill; Principles of Process Planning, $1^{\text {a }}$ edición. 317-332, Chapmann \& Hall, UK (1995).

Holland J.H.;Adaptation in Natural and Artificial Systems, 90-110, MIT Press, (1992).

Kaldor, S. y P.K. Venuvinod; Macro level Optimization of Cutting Tool Geometry. J. of Mfg. Sci. and Eng.: Feb., 119(1-9) (1997).

Kalpakjian, S. y S.R. Schmid; Manufactura, Ingeniería y Tecnología, 4ª edición, 25-26, Pearson Education, México (2002).

McMullen, P.R., M. Clark, D. Albritton y J. Bell; A correlation and heuristic approach for obtaining production sequences requiring a minimum of tool replacements, Int. J. Comput. Oper. Res.: 30(3), 443-462 (2003).

Oral A. y M.C. Cakir; Automated cutting tool selection and cutting tool sequence optimisation for rotational parts, Robotics and Computer-Integrated Manufacturing, 20(2), 127-141 (2004).

Quiza, R., M. Rivas y E. Brindis, Genetic algorithm-based multi-objective optimization of cutting parameters in turning processes, Eng. App. Art. Int.: 19(7),127-133 (2006)

Stemmer, C.E; Ferramentas de Corte I, 4º edición, 61-64, Editora UFSC, Brasil, (1995).

Toussaint, J. y K. Cheng, Web-based CBR (case-based reasoning) as a tool with the application to tooling selection Int J. Adv Manuf. Technol.: 29(1-2), 24-34 (2006).

Zhao, Y., K. Ridgway y K, A.M.A., Al-Ahmari; Integration of CAD and a cutting tool selection system Computers and Industrial Engineering archive: 42(1), 17-34 (2002). 